\title{
Changes of endogenous hormones during ovary development in Rhododendron delavayi $\times$ Rhododendron sinofalconeri and Rhododendron delavayi $\times$ Rhododendron cyanocarpum
}

\author{
A.A. El-Tantawy ${ }^{1,2}$, Weijia Xie ${ }^{1,3}$, Shifeng $\mathrm{Li}^{1}$, Jihua Wang ${ }^{1}$, Lvchun Peng ${ }^{1}$, Jie Song ${ }^{1}$, Lu Zhang ${ }^{1}$, Yanfei Cai ${ }^{1}$ \\ and Xiumei Yang ${ }^{1}$ \\ ${ }^{1}$ Flower Research Institute, Yunnan Academy of Agricultural Sciences, Kunming, China \\ ${ }^{2}$ Ornamental Horticulture Department, Faculty of Agriculture, Cairo University, Egypt \\ ${ }^{3}$ School of Life Sciences, Center for Plant Disease \& Pest Biocontrol, Yunnan University, Kunming, China
}

\begin{abstract}
Summary
Endogenous plant hormones (EPHs) play a key role in plant development coordination, including floral bud development and fruit growth. Studying of EPHs during ovary development is very important to understand the growth regulation in rhododendron, which is the most important economic ornamental woody plant in the world. In this study, cross-pollination was performed between Rhododendron delavayi as female parent with $R$. cyanocarpum and $R$. sinofalconeri as male parents. Concentrations of different EPHs were evaluated in ovary development during days after cross-pollination (DAPs) using the HPCL technique. Jasmonic acid (JA) and jasmonoyl-L-isoleucine (JA-Ile) content recorded as the highest endogenous hormone level suggesting the low temperature stress faced by the plants during their growth in the forest special during winter season (January). Concentrations of Indole-3-acetic acid (IAA) peaked on 25 DAPs in both species and decreased during further days. The ovary consisted of the highest abscisic acid (ABA) level on the cross-pollination day (0 DAPs) indicating its inhibition effect on Rhododendron fruit growth. The highest level of $\mathrm{GA}_{9}, \mathrm{GA}_{19}$ and $\mathrm{GA}_{4}$ was recorded on 17 DAPs whereas $\mathrm{GA}_{4}, \mathrm{GA}_{44}$ and $\mathrm{GA}_{20}$ contents indicated that GAs has an important role in ovary development for both studied species. In conclusion, although no significant differences were found in the ovary measurements between both species, highly significant variations were recorded in EPHs changes after cross-pollination indicating the influence of the two male parents' genotypes. Our results in EPHs changes during ovary development opened a gate to find a new modern way for saving the rare Rhododendron plants.
\end{abstract}

Keywords

ABA, cross-pollination, endogenous hormones, GAs, IAA, JA, Rhododendron, SA, ZA

\section{Introduction}

Endogenous plant hormones (EPHs) are a group of molecules that are secreted naturally within almost all the plant organ tissues by secondary metabolism (Santner and Estelle, 2009). EPHs play a key role in plant development as

\section{Significance of this study}

What is already known on this subject?

- Rhododendron is the most economic ornamental woody plant in the world. Studying the growth development is very important for the conservation.

What are the new findings?

- Our research indicated the significant differences in EPHs changes during the ovary development influenced by the two male parents' genotype.

What is the expected impact on the horticulture?

- This has opened a gate to find a new modern way for saving the rare Rhododendron plants and its breeding.

they regulate cell division, proliferation and differentiation (Perrot-Rechenmann, 2010). Most of these hormones can stimulate the adventitious root (Sang et al., 2016), leaf abscission (Sauer et al., 2013), seed development (Koornneef et al., 1984), and zygotic embryos (Reinhardt et al., 2000).

Since the last decade, new insights in the EPHs actions during fruit growth, set and ripening have been indicated (Kumar et al., 2014). Also, EPHs play an important role in ovary increment due to its ability in the regulation of epidermal cell elongation in the ovary (Zhang et al., 2016). Several reports studied the role of EPHs during fruit development in the woody species (Liu et al. [2018] in pear; Zhou et al. [2015] in chestnut). In parallel, studies on EPHs during zygotic embryogenesis (Robert et al., 2015), somatic embryogenesis (Su et al., 2014), and microspore embryogenesis (Rodríguez-Sanz et al., 2014), as well as their role in seed development (Barendse et al., 1991) covered many aspects in the hormone changes during the flowering plant development in general. However, knowledge of the regulation of EPHs during ovary development is still scarce because of the mechanism complexity of the hormone controlling the developing fruit (Pattison et al., 2014; Kumar et al., 2014), which shows difficulties in building an ideal data on plant hormones during ovary development.

Rhododendron belongs to the family Ericaceae and the subfamily Rhododendroideae; it is one of the largest genera encompassing more than 1,000 species around the world (Chamberlain et al., 1996). More than 600 species have been found in China, especially in Yunnan and Tibet where the first 
record of Rhododendron presence has been acknowledged (De Milleville, 2002). Studying the Rhododendron growth development is very important for the conservation of the biological balance in surrounding ecosystems (Bharali and Latif Khan, 2012). Therefore, investigation into the changes of EPHs levels during ovary development in Rhododendron is needed. Nevertheless, changes in EPHs levels during ovary growth in Rhododendron have not been elucidated.

In this work, changes of the EPHs contents (jasmonates/abscisic acid ABA/indole-3-acetic acid IAA/zeatin ZA/ gibberellins GAs) were studied during ovary development in $R$. delavayi after cross pollination with the extremely endangered species, $R$. cyanocarpum, and the most threatened species, $R$. sinofalconeri, using high performance liquid chromatography (HPLC), along with the measurement of the ovary width and length in the same studied species. Due to the limitation in use of Rhododendron resources, the study of distant hybridization barriers in this important genus is highly required, as new varieties that tolerate the environmental stress could be produced. Natural hybridization between $R$. delavayi and $R$. cyanocarpum subgenus Hymenanthes in Yunnan, China, has been detected (Ma et al., 2010). Also, cross pollination between $R$. delavayi and some other species like $R$. decorum (Zha et al., 2008) and $R$. irroratum (Zha et al., 2010) has also been observed, indicating variation in fruit set among pollination treatments. Although hybridization among some Rhododendron species has been observed by morphological and molecular marker methods (Kita et al., 2005), the role of hybridization in ovary and fruit development is still unclear. Cross pollination produced higher sets of seeds in some species of Rhododendron compared with self-pollination (Ng and Corlett, 2000). As high concentrations of EPHs are also essential for fruit set in many plant species including cross pollinated plants (De Jong et al., 2006), our research presents the first comparative study between the studied species in respect of changes in EPHs after cross pollination as a key factor in a classification among Rhododendron species. The main goal of our research is to open a new gate in Rhododendron breeding through detecting the differences among the most economic species by showing the relationship between EPHs contents and ovary development, in order to find several solution methods for threatened rhododendrons in China and around the world.

\section{Materials and methods}

\section{Study location}

The study area was located in Kunming Jidian Forest Nature Center $\left(25^{\circ} 04^{\prime}-25^{\circ} 11^{\prime} \mathrm{N}, 102^{\circ} 43^{\prime}-102^{\circ} 53^{\prime} \mathrm{E}\right)$, which is in the northeastern suburb of Kunming. It belongs to the subtropical southwest monsoon climate, with distinct dry and wet seasons. The annual average temperature is $11.2 \sim 13.8^{\circ} \mathrm{C}$, the monthly temperature is the highest in July which is $18.2^{\circ} \mathrm{C}$, the monthly minimum temperature (January) is $6.8^{\circ} \mathrm{C}$; the annual average precipitation is $980.5 \sim 1,156 \mathrm{~mm}$, and the annual rainfall is concentrated from May to August.

\section{Materials}

The plant of $R$. delavayi in the blooming period, which flowers from February to April in Kunming Jidian Forest Nature Center, was used as a female parent. $R$. cyanocarpum and $R$. sinofalconeri were used as male parents, respectively. The pollens of $R$. cyanocarpum were collected from the Hudianba of Cang Mountain in Dali, Yunnan, which flowers from March to May at an altitude of 3,200 m. The pollens of $R$. sinofal- coneri were collected from the Bozhu Peak of Laojun Mountain in Wenshan, Yunnan, which flowers from April to May at an altitude of 2,900 $\mathrm{m}$. The pollens were collected from the anther and dried for $24-48 \mathrm{~h}$, and then stored at $-80^{\circ} \mathrm{C}$ for storage.

\section{Pollen viability}

Before pollination, the stored pollens of $R$. cyanocarpum and $R$. sinofalconeri were transferred to a 12 -well plate and submerged in $500 \mu \mathrm{L}$ liquid germination medium according to Tian and Russell (1997). The medium consisted of $(10 \%$ (w/v) sucrose, $0.01 \%(\mathrm{w} / \mathrm{v}) \mathrm{H}_{3} \mathrm{BO}_{3}, 0.01 \%(\mathrm{w} / \mathrm{v}) \mathrm{CaCl}_{2}$, $0.02 \%(\mathrm{w} / \mathrm{v}) \mathrm{MgSO}_{4} \cdot 7 \mathrm{H}_{2} \mathrm{O}$, and $0.01 \%(\mathrm{w} / \mathrm{v}) \mathrm{KH}_{2} \mathrm{PO}_{4}$. The pollen viability of $R$. cyanocarpum and $R$. sinofalconeri was $57.68 \pm 4.34 \%$ and $45.52 \pm 4.12 \%$, respectively, which is suitable for pollination.

\section{Pollination time and method}

The crosses were conducted from March $15^{\text {th }}$ to April $15^{\text {th }}, 2018$, with $R$. delavayi closed flowers during pre-anthesis stage as the female and $R$. cyanocarpum or $R$. sinofalconeri as the male, respectively. Sulfated paper bags were used in the pollination and pushed away at 1 week after pollination.

\section{The ovary measurements}

Ten ovaries from 10 different inflorescences of $R$. delavayi $\times R$. cyanocarpum or $R$. delavayi $\times R$. sinofalconeri were harvested at $0,17,25,33,40$, and 47 days after the pollination. Both ovary width and length was measured using a digital caliper.

\section{The hormone content determination}

At least twenty ovaries from 10 different inflorescences of $R$. delavayi $\times R$. cyanocarpum or $R$. delavayi $\times R$. sinofalconeri were harvested at $0,17,25,33,40$, and 47 days after pollination, followed by immediate placement into an ice box. The samples were weighted to $0.5 \mathrm{~g}$ in the cool conditions at the laboratory; they were then bagged in aluminum foil and submerged in liquid nitrogen, and stored at $-80^{\circ} \mathrm{C}$ for storage.

Extraction of IAA, ABA, ZA, and GA was done according to Ergun and Topcuoglu (2002). $0.5 \mathrm{~g}$ of frozen ovary samples were homogenized in an ice bath. After that, the grinded samples were immersed in $10 \mathrm{~mL}$ cold $80 \%$ (v/v) methanol, then incubated at $4^{\circ} \mathrm{C}$ for $24 \mathrm{~h}$. The samples were centrifuged at $12,000 \mathrm{rpm}$ for $15 \mathrm{~min}$ at $4^{\circ} \mathrm{C}$ to discard any particulates. Two drops of ammonia were added to the samples, they were reduced to half by an evaporator followed by adding two drops of ultrapure water (Mili Q). IAA, ABA, and GA were extracted with equal volumes of ethyl acetate ( $\mathrm{pH} 2-3)$. The samples were then desiccated at $40^{\circ} \mathrm{C}$ after adding two drops of ammonia followed by storing at $-20^{\circ} \mathrm{C}$. For ZA extraction, pH was adjusted to 7.5 with $2 \mathrm{~N} \mathrm{NaOH}$ and extracted with an equal volume of saturated $\mathrm{N}$-butanol. The samples were then dried at $60^{\circ} \mathrm{C}$ after adding two drops of ammonia followed by storing at $-20^{\circ} \mathrm{C}$.

\section{HPLC determination}

High performance liquid chromatography (HPLC) was used to determine the concentrations of IAA, ABA, ZA, and GA as previously described by Ding et al. (2006). $5 \mu \mathrm{L}$ samples were injected into the column at $30^{\circ} \mathrm{C}$ with a flow rate $1.0 \mathrm{~mL} \mathrm{~min}^{-1}$. To detect IAA, ABA and GA, the mobile phase was methanol and glacial acetic acid $(40: 60, v / v)$, whereas methanol with ultrapure water $(40: 60, v / v)$ was used to de- 
tect ZA. Histograms were conducted to quantify the endogenous plant hormones (EPHs) extracted from un-pollinated and pollinated plants.

\section{Statistical analyses}

10 fruits were randomly taken for the required measurement. Data were analyzed according to Demsar et al. (2013), using SPSS v.22 software by variance of differences (ANOVA) test. The degree of freedom was followed as $P \leq 0.05$ and $P \leq 0.01$ considers statistical significance and represents as *, ** for comparison between MS and MC and \#, \#\# for comparison among DAPs, respectively. Standard Error (SE) was estimated using the values of the Standard Deviation (SD) using this formula: $\mathrm{SE}=\mathrm{SD} / \sqrt{\mathrm{n}}$. Data are shown as mean $\pm \mathrm{SE}$ of different replicates.

\section{Results}

\section{Growth of ovary during days after cross-pollination}

Changes in ovary growth of $R$. delavayi $\times R$. sinofalconeri $(M S)$ and $R$. delavayi $\times R$. cyanocarpum $(M C)$ are shown in Figure 1. In both species, gradual increase in width and length was observed as shown in Figure 1. Significant fast growth was observed started from the fruit initial day (17 DAPs) until 47 DAPs. Also, we observed that no significant differences between $M S$ and $M C$ in ovary width, except for the level on 33 DAPs in which a significant difference between our cross-pollinated species was recorded (Figure 1A). Similar-

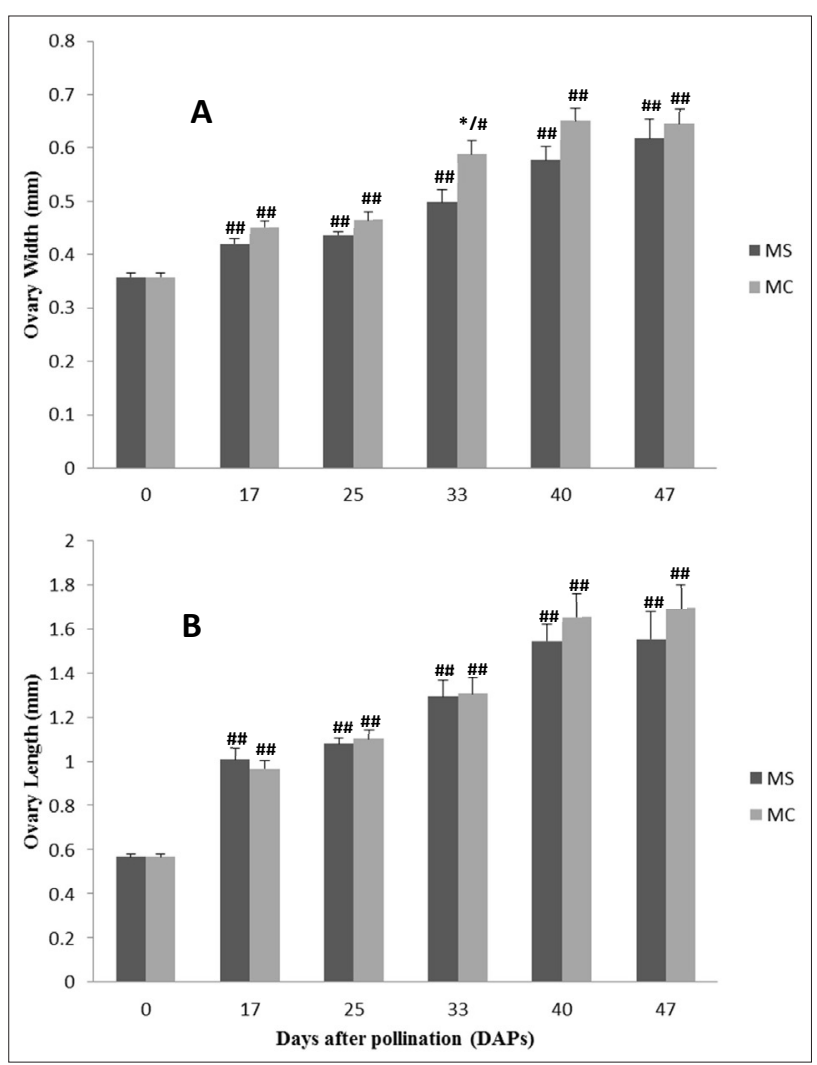

FIGURE 1. Ovary growth of Rhododendron delavayi $\times$ R. sinofalconeri $(M S)$ and $R$. delavayi $\times R$. cyanocarpum $(M C)$ during days after pollination (DAPs). (A) ovary width; (B) ovary length. Data show mean \pm SE of the 10 replicates. * ** indicate significant differences with $M S$ and $M C$ at $\mathrm{P} \leq 0.05$ or 0.001 , respectively, whereas \#, \#\# indicate significant variations by DAPs versus 0 DAPs at $\mathrm{P} \leq 0.05$ or 0.001 , respectively. ly, a significant increase was observed in ovary length from 17 DAPs to 47 DAPs (Figure 1B).

\section{Changes in endogenous hormones during ovary development}

1. Jasmonate (JA and JA-Ile). Jasmonate contents during ovary development in both $M S$ and $M C$ were attentive; we evaluated the level of JA and JA-Ile (concentrations are shown in Figure 2). The contents of JA during ovary growth in $M S$ were relative high on 0 DAPs and peaked at 17 DAPs (Figure 2A). It was then followed with notable low levels at 25 DAPs and reached after gradual decrease the lowest concentration on 47 DAPs when there is a significant difference compared with 0 DAP (Figure 2A). On the contrary, JA concentration level was highest before fruit initiation (on 0 DAPs), followed by a sudden decline on fruit initial day (17 DAPs) in $M C$. From 17 to 25 DAPs, a slight increase of JA content was observed in the same species (Figure 2A). Further, gradual decrease was recorded from 33 to 47 DAPs (Figure 2A). Highly significant difference was observed in 33 DAP in comparison with 0 DAP.

As in JA content, the highest level of JA-Ile was recorded at 17 DAPs in $M S$ (Figure 2B). After that, the content notably decreased to the lowest levels at 47 DAPs (Figure 2B). In $M C$, the ovary consisted of a slight high concentration of JA-Ile on

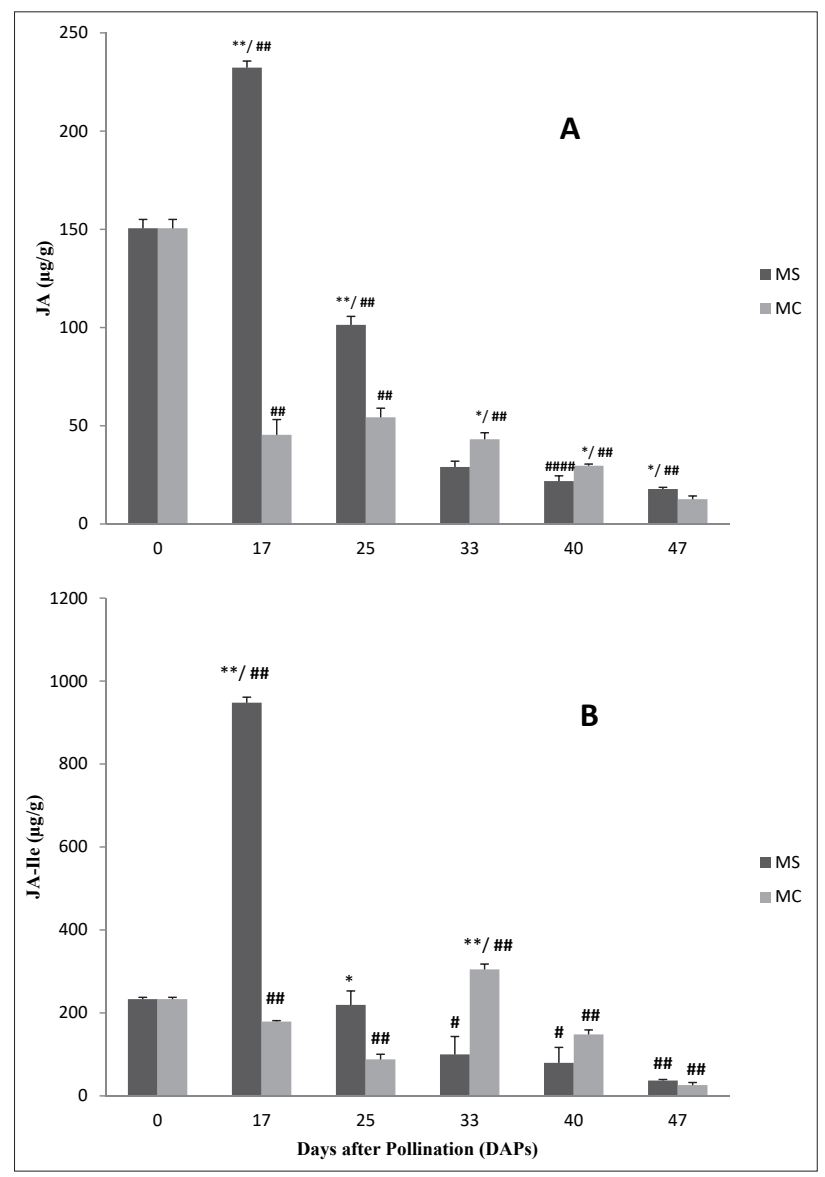

Figure 2. Changes in endogenous (A) JA and (B) JA-Ile contents in the ovary of Rhododendron delavayi $\times R$. sinofalconeri $(M S)$ and $R$. delavayi $\times R$. cyanocarpum $(M C)$ during days after pollination (DAPs). Data show mean \pm SE of the 3 replicates. *, ** indicate significant differences with $M S$ and $M C$ at $\mathrm{P} \leq 0.05$ or 0.001 , respectively, whereas \#, \#\# indicate significant variations by DAPs versus 0 DAPs at $\mathrm{P} \leq 0.05$ or 0.001 , respectively. 


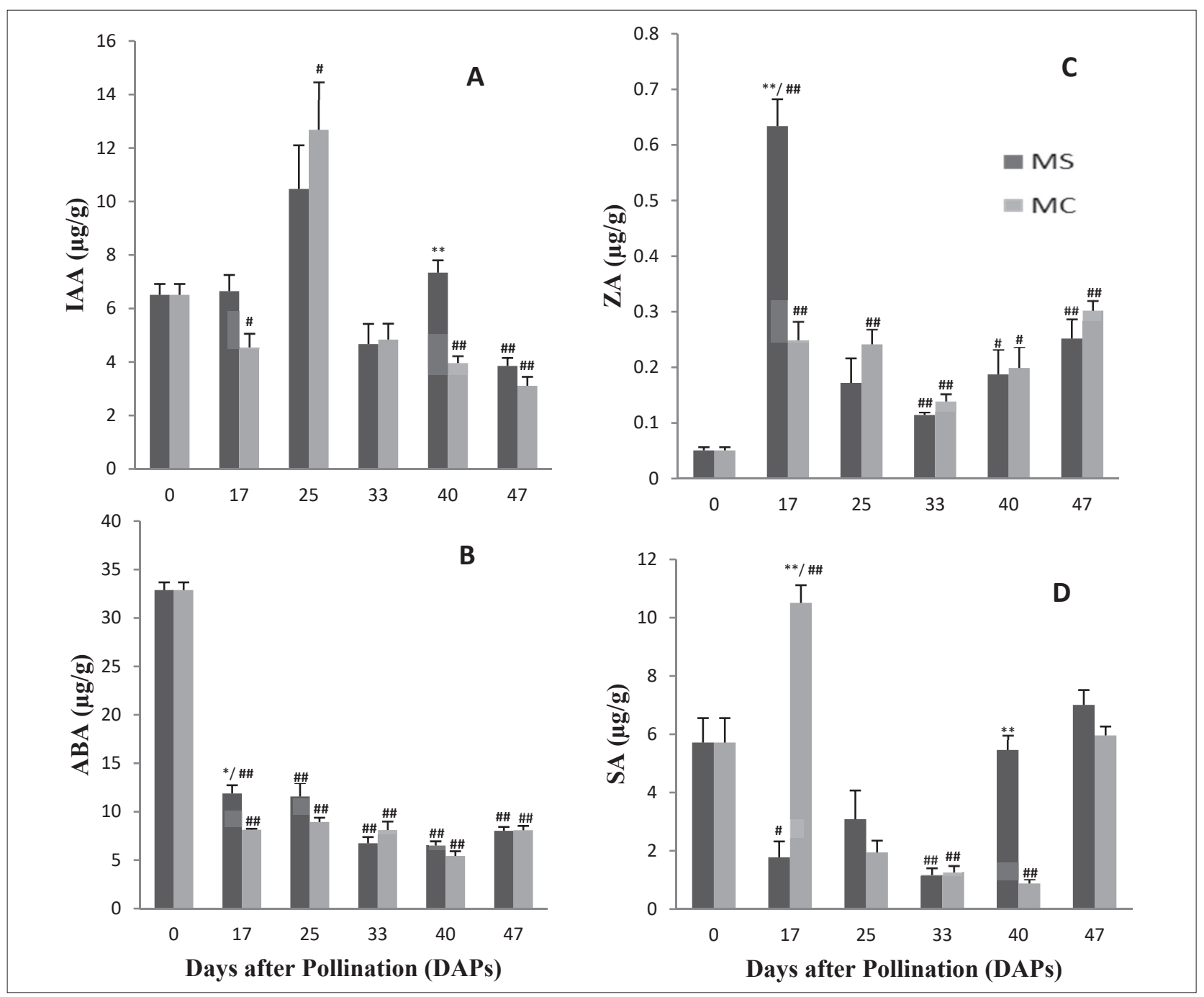

Figure 3. Changes in endogenous (A) IAA, (B) ABA, (C) ZA, and (D) SA contents in the ovary of Rhododendron delavayi $\times$ $R$. sinofalconeri $(M S)$ and $R$. delavayi $\times R$. cyanocarpum $(M C)$ during days after pollination (DAPs). Data show mean $\pm \mathrm{SE}$ of the 3 replicates. *, ** indicate significant differences with $M S$ and $M C$ at $\mathrm{P} \leq 0.05$ or 0.001 , respectively, whereas \#, \#\# indicate significant variations by DAPs versus 0 DAPs at $\mathrm{P} \leq 0.05$ or 0.001 , respectively.

cross pollination day (0 DAPs), it was followed by a gradual decline to a relative increase starting from 25 DAPs, peaked significantly at 33 DAPs and decreased again reaching the minimum level at 47 DAPs (Figure 2B).

2. IAA, ABA, ZA, and SA contents. Changes in endogenous IAA, ABA, ZA and SA contents in $M S$ and $M C$ are shown in Figure 3. In $M S$, we observed a slight increase in IAA concentration on 17 DAPs compared with the pollination day (0 DAPs), whereas a significant decrease was found from 0 to 17 DAPs in $M C$ (Figure 3A). Therefore, notable increase up to a peak was observed at 25 DAPs, followed by a significant decrease from 33 DAPs to 47 DAPs in both species (Figure 3A). It was noticeable that only IAA content increased significantly in $M S$ from 33 to 40 DAPs causing a highly significant difference with $M C$ on 40 DAPs (Figure 3A). ABA concentration was at peak on the cross-pollination day (0 DAPs) in both species, then, a notable decline during ovary growth in both species (Figure 3B).

ZA content was very low on 0 DAPs (Figure 3C). In MS, the highest ZA concentration was notably recorded on 17 DAPs followed by a significant decrease from 17 to 33 DAPs. Then, a slight increase was observed from 33 to 47 DAPs (Figure 3C). While in MC, the peak was found on 47 DAPs after decreasing from 17 to 33 DAPs with a gradual increase from
33 to 47 DAPs (Figure 3C). It is worthy to note that there is a highly significant difference between the two cross-pollinated fruits on 17 DAPs, which was not found until 47 DAPs (Figure 3C).

In SA content, it was observed that there was a very high significant variation during ovary growth in both plants. In $M C$, the content was a little high on pollination day and a sudden peak was observed on 17 DAPs, followed by a notable high decrease until 40 DAPs; then the content was increased again on 47 DAPs (Figure3D). In $M S$, the concentration was also of a very low level, starting on 17 DAPs with a slight increase on 33 DAPs followed by a highly significant increase on 40 DAPs, reaching to the maximum level on 47 DAPs (Figure 3D).

3. Gibberellins (GAs). Due to the importance of endogenous gibberellins (GAs) during ovary development in the angiosperms generally and in the woody plants in particular, nine types of GA family were studied during ovary growth in $M S$ and $M C$. Changes in concentrations of endogenous $\mathrm{GA}_{7}$, $\mathrm{GA}_{3}$, and $\mathrm{GA}_{53}$ are presented in Figure 4. In $M S$, the maximum high level in $\mathrm{GA}_{7}$ content was recorded on 0 DAPs which significantly decreased on 17 DAPs to 47 DAPs (Figure 4A). While in $M C$, a slightly high content of $\mathrm{GA}_{7}$ was observed on the cross-pollination day (0 DAPs) which decreased on 


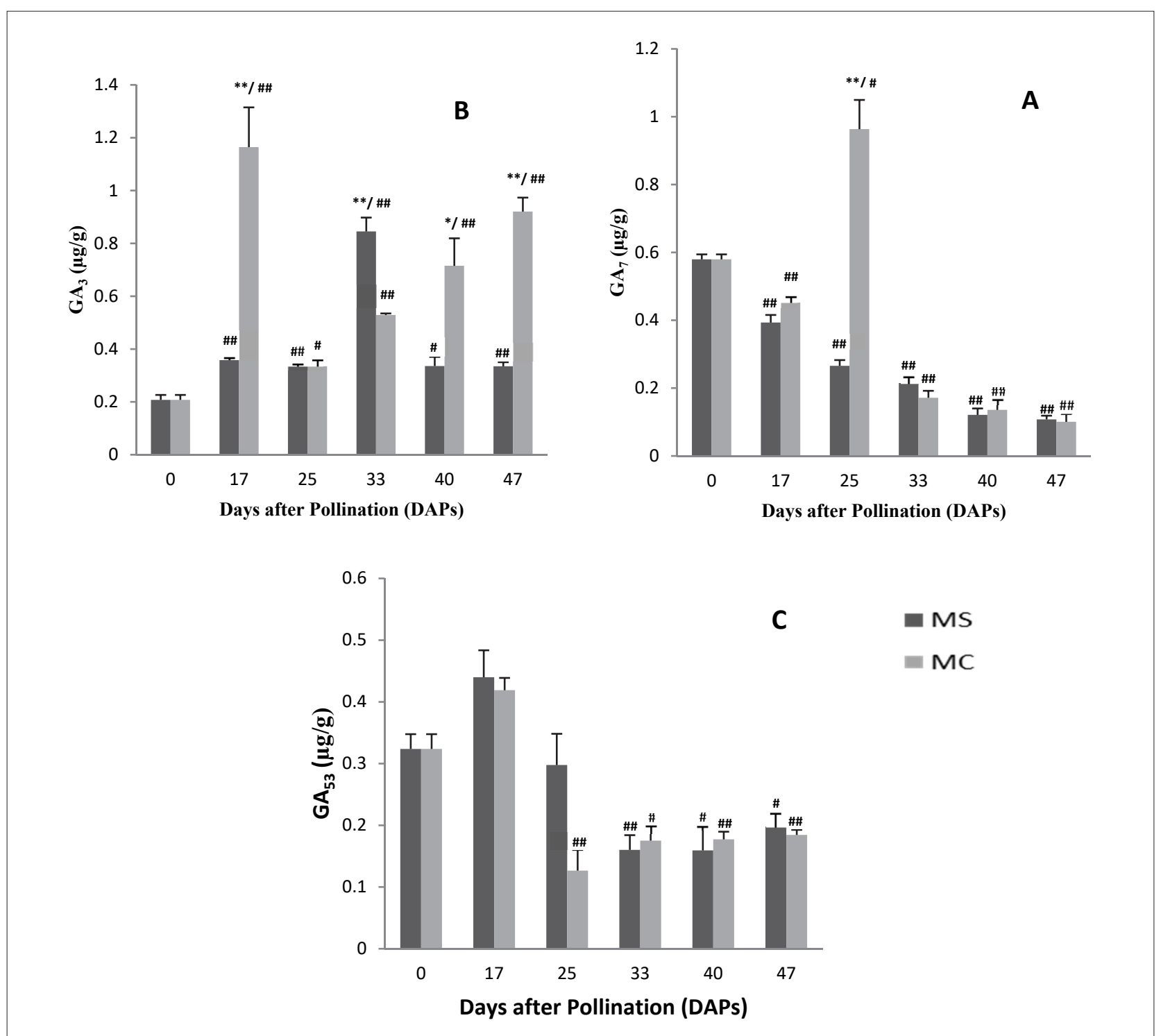

FigurE 4. Changes in endogenous (A) GA3, (B) GA7, and (C) GA53 contents in the ovary of Rhododendron delavayi $\times$ R. sinofalconeri $(M S)$ and $R$. delavayi $\times R$. cyanocarpum $(M C)$ during days after pollination (DAPs). Data show mean \pm SE of the 3 replicates. ${ }^{*}, * *$ indicate significant differences with $M S$ and $M C$ at $\mathrm{P} \leq 0.05$ or 0.001 , respectively, whereas \#, \#\# indicate significant variations by DAPs versus 0 DAPs at $\mathrm{P} \leq 0.05$ or 0.001 , respectively.

17 DAPs (Figure 4A). Then, it was followed by a notable increase to a peak on 25 DAPs; a second decrease was noted from 25 to 47 DAPs (Figure 4A).

Regarding $\mathrm{GA}_{3}$ content, gradual increase was observed from 0 DAPs which reached notably to a peak on 33 DAPs for $M S$ followed by reduction in the concentration on 40 and 47 DAPs (Figure 4B). For $M C$, it peaked on 17 DAPs followed by decrease in $\mathrm{GA}_{3}$ content on 25 DAPs and peaked again on 47 DAPs (Figure 4B). During the whole ovary growth in both species, $\mathrm{GA}_{3}$ levels in $M S$ were lower than in $M C$ on 17,40 , and 47 DAPs, in which significant differences were found between the two studied species; whereas the level in $\mathrm{GA}_{3}$ content of $M S$ was significantly higher (Figure 4B). The highest concentrations of $\mathrm{GA}_{53}$ during ovary growth in the two species were recorded on 17 DAPs, followed by a significant decrease from 25 to 47 DAPs (Figure 4C). Worthy to note that $\mathrm{GA}_{53}$ content was significantly higher than in $M C$ on 25 DAPs while no significant differences were found until 47 DAPs (Figure 4C).

Figure 5 presented the concentrations of $\mathrm{GA}_{20}$ and $\mathrm{GA}_{9}$ during ovary development in $M S$ and $M C$. Increase of $\mathrm{GA}_{20}$ in the two species from 0 to 17 DAPs (Figure 5A). From 17 to 25 DAPs, slight increase was found in $M C$, with a sudden decline in $M S$ causing a highly significant difference between both species (Figure 5A). Then, a notable increase was found and peaked on 40 and 47 DAPs for $M C$ and $M S$, respectively (Figure 5A). Highly significant differences between $M C$ and $M S$ were found, as a very low level was recorded on 47 DAPs in $M C$ (Figure 5A). The maximum level of $\mathrm{GA}_{9}$ content was measured on 17 DAPs in $M C$, while it reached the highest level on 25 DAPs in MS (Figure 5B). Gradual decreases and increases were recorded in $M S$ and $M C$, respectively, starting from 25 DAPs to 40 DAPs (Figure 5B). From 40 to 47 DAPs, slight increase in $\mathrm{GA}_{9}$ level was observed in $M S$ while significant increase was observed in $M C$ (Figure 5B).

Regarding the concentrations of $\mathrm{GA}_{19}, M S$ contained a high level of $\mathrm{GA}_{19}$ during the early ovary development compared with $M C$ which peaked at 17 DAPs, followed by a gradual decrease until 47 DAPs for MS (Figure 6A). For $M C$, a maximum level of $\mathrm{GA}_{19}$ content was recorded at 0 DAPs which was followed by gradual decrease until 33 DAPs; it increased again on 40 DAPs followed by decreasing to the same level 


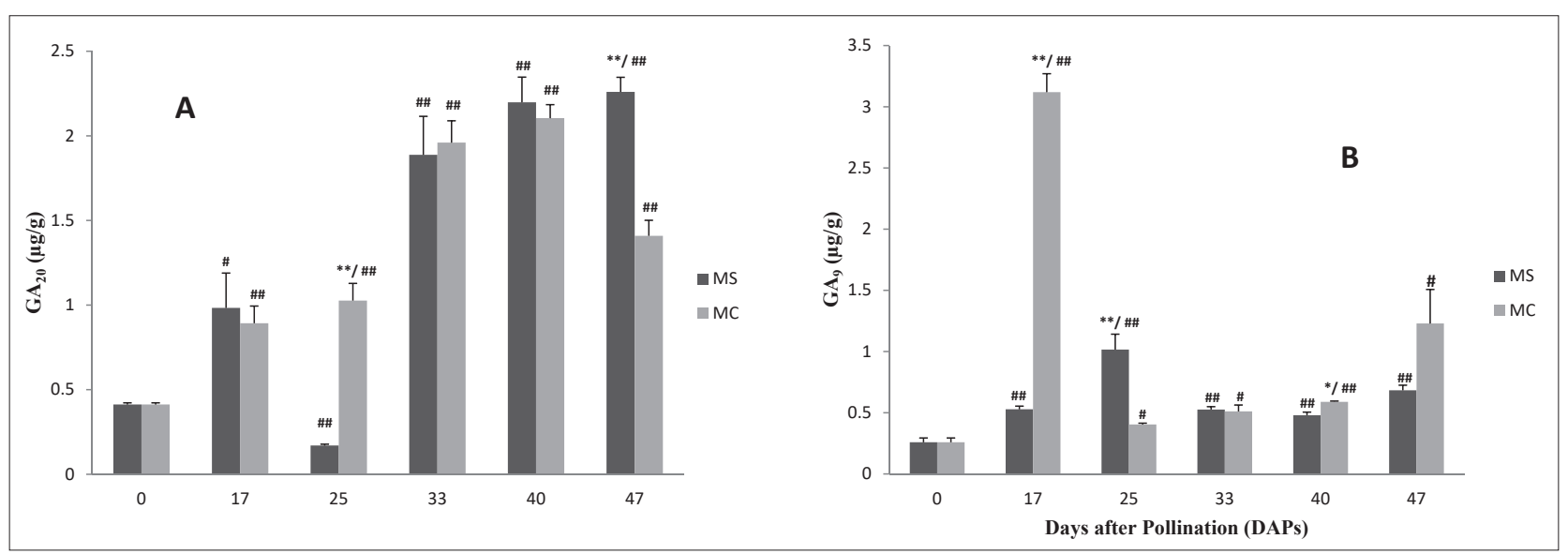

FIGURE 5. Changes in endogenous (A) GA20 and (B) GA9 contents in the ovary of Rhododendron delavayi $\times$ R. sinofalconeri $(M S)$ and $R$. delavayi $\times R$. cyanocarpum $(M C)$ during days after pollination (DAPs). Data show mean \pm SE of the 3 replicates. *,** indicate significant differences with $M S$ and $M C$ at $\mathrm{P} \leq 0.05$ or 0.001 , respectively, whereas \#, \#\# indicate significant variations by DAPs versus 0 DAPs at $\mathrm{P} \leq 0.05$ or 0.001 , respectively.

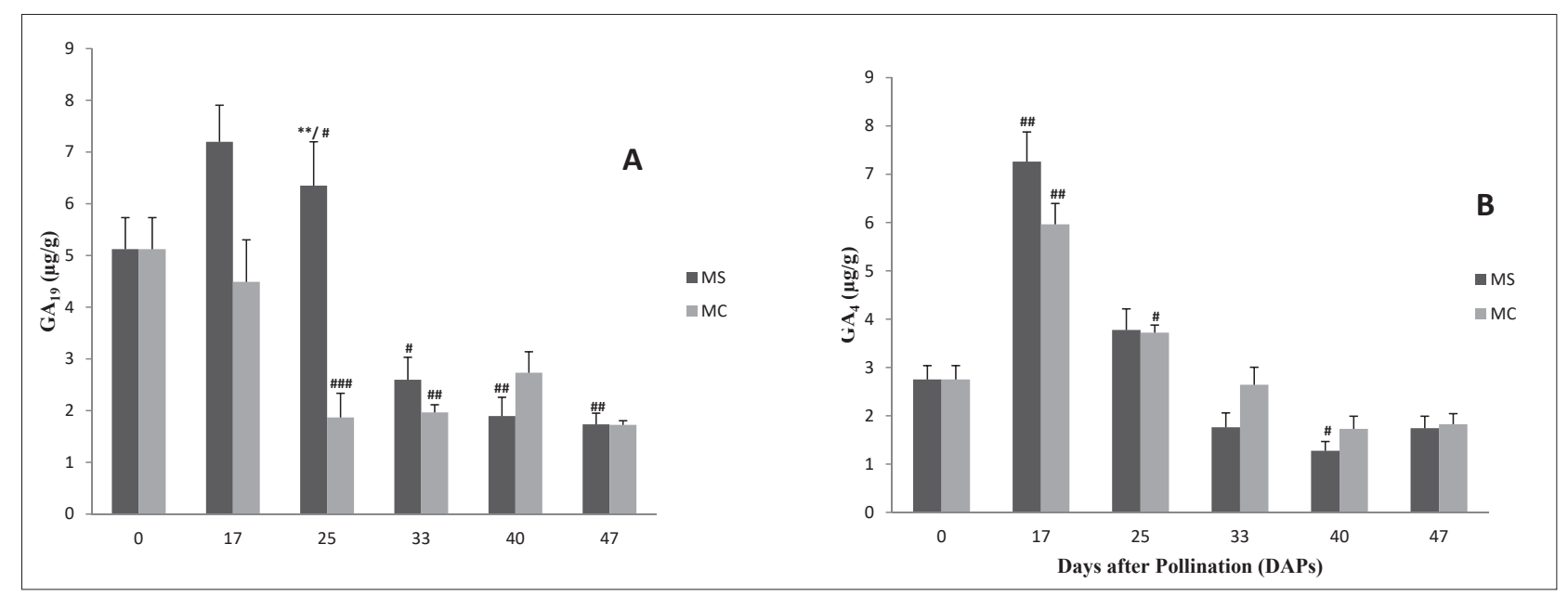

FIGURE 6. Changes in endogenous (A) GA19 and (B) GA4 contents in the ovary of Rhododendron delavayi $\times$ R. sinofalconeri $(M S)$ and $R$. delavayi $\times R$. cyanocarpum $(M C)$ during days after pollination (DAPs). Data shown mean \pm SE of the 3 replicates. *, ** indicate significant differences with $M S$ and $M C$ at $\mathrm{P} \leq 0.05$ or 0.001 , respectively, whereas \#, \#\# indicate significant variations by DAPs versus 0 DAPs at $\mathrm{P} \leq 0.05$ or 0.001 , respectively.

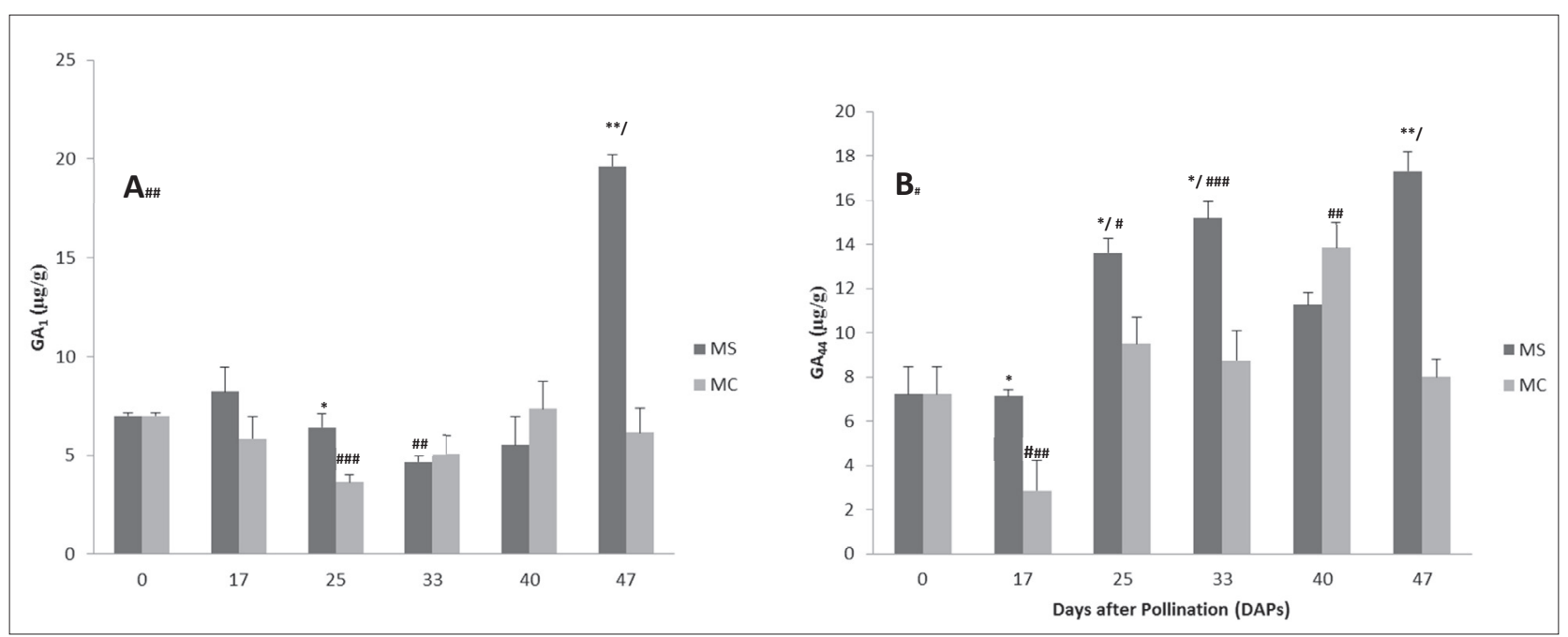

FigurE 7. Changes in endogenous (A) GA1 and (B) GA44 contents in the ovary of Rhododendron delavayi $\times$ R. sinofalconeri $(M S)$ and $R$. delavayi $\times R$. cyanocarpum $(M C)$ during days after pollination (DAPs). Data show mean \pm SE of the 3 replicates. *, ** indicate significant differences with $M S$ and $M C$ at $\mathrm{P} \leq 0.05$ or 0.001 , respectively, whereas \#, \#\# indicate significant variations by DAPs versus 0 DAPs at $\mathrm{P} \leq 0.05$ or 0.001 , respectively. 


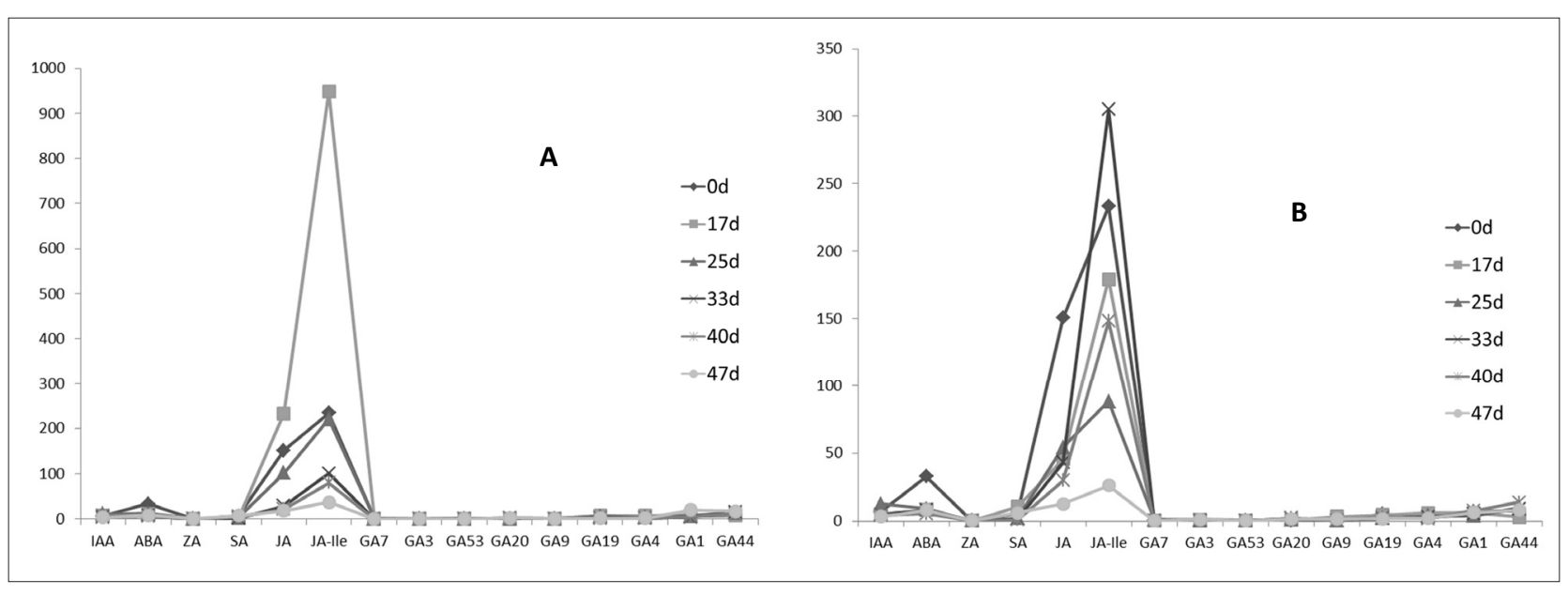

FIGURE 8. Differences among the studied endogenous hormones during the days of ovary development after cross-pollination (d) in the ovary of (A) Rhododendron delavayi $\times$ R. sinofalconeri and (B) R. delavayi $\times$ R. cyanocarpum.

of $M S$ at 47 DAPs (Figure 6A). $\mathrm{GA}_{4}$ concentrations increased notably to a maximum level on 17 DAPs, followed by a significant decrease starting from 17 DAPs; and a gradual decrease was recorded again on 47 DAPs (Figure 6B). Finally, a slight increase was found in $\mathrm{GA}_{1}$ content on 17 DAPs in $M S$ and $M C$ (Figure 7A). The concentration in both species reached a similar level on 33 DAPs. On 47 DAPs, it peaked for $M S$ and significantly decreased for $M C$ (Figure 7A). $\mathrm{GA}_{44}$ levels were significantly higher in $M S$ compared with $M C$ from 17 DAPs to 33 DAPs (Figure 7B). On the contrary, a maximum level was recorded on 40 DAPs, followed by a significant decline for $M C$ whereas in $M S$, the level reached to a peak on 47 DAPs (Figure 7B).

\section{Differences among endogenous hormones during ovary development after cross-pollination}

Figure 8 identified the differences among the studied hormones. We found, in both species, that JA-Ile recorded the highest level during ovary growth after pollination in comparison with the other endogenous hormones. Moreover, JA content recorded the second-highest level, slight differences were found with JA-Ile on all DAPs except for the measurement on 17 DAPs and 33 DAPs, in which JA-Ile level was notably higher in $M S$ and $M C$, respectively. On the other hand, we observed that ABA was recorded as the third-highest level on 0 DAPs, followed by GA44 on 40 DAPs in both two plants.

\section{Discussion}

Generally, cross-pollination is effective on fruit set and morphological characteristics in flowering plants (Yildiz and Kaplankiran, 2017). Investigation on cross-pollination among the economic plant species has been conducted to study some important physiological problems such as self-incompatibility (Yildiz and Kaplankiran, 2017) and threats facing woody plants, both by natural disasters or population density (Gram and Sork, 1999). In Rhododendron, cross-pollination has been mainly used as a key factor in speciation (Zhang et al., 2007). It has been indicated that $R$. delavayi has a low level of self-compatibility, leading to interest in investigating natural and artificial cross-pollination with other Rhododendron species (Zhang et al., 2007). In this work, we used $R$. delavayi as a female plant to cross pollinate with $R$. cyanocarpum and $R$. sinofalconeri as male plants. The fruit growth was surveyed and the changes of ovaries in the critical period of the different embryo development phases were compared on different days. Fruit initial days started from 17 to 47 DAPs which is considered a fast rate in ovary growth, indicating the early embryo development stage as described by Xie et al. (2016) in R. decorum $\times R$. irroratum.

Regarding the changes of EPHs during ovary development, we performed a comparative study between $M S$ and $M C$. Data presented in this work indicate that jasmonate content during ovary growth plays a key role in fruit initiation in $M S$ and $M C$; we also found that the concentrations of the other studied hormones are negligible compared to JA and JA-Ile contents. Jasmonate has mainly been found in jasmine plants (Baldwin et al., 2006). Mainly, jasmonate plays an essential role in the response of plants to abiotic and biotic stresses as well as plant development such as senescence, flower abscission (Oh et al., 2013). In this respect, the low temperature stresses faced by rhododendron in the forest may explain the notable high concentrations of JA and JA-Ile during ovary development. Although SA contents were at low levels, their presence is very important due to the antagonistic relationship between SA and jasmonic acid (Ballaré, 2011), which has found that their role is in pathogen defense in rice (Tamaoki et al., 2013). Moreover, it has been shown that jasmonic acid induced resistance against broad mites in R. semsii (Luypaert et al., 2017). Besides, it has been indicated that jasmonic acid was a very high content during the early stages in apple and cherry fruits, and was followed by a decline in the days after blooming and increased again during fruit maturity (Kondo and Tomiyama, 2000). Similar results were found in this work during ovary development, indicating the role of JA and JA-Ile in the early stages in $M S$ and $M C$ fruit formation.

Results of changes in IAA and ABA levels showed almost similar patterns between both two species. IAA concentration level peaked on 25 DAPs in both species, followed by decreasing during ovary development. While, the maximum level of ABA was on 0 DAPs which decreased notably during ovary development. Probably this result is related to the rapid ovary growth starting from 25 DAPs, which has indicated that IAA plays an important role in fruit cell division and ovary development (Zou et al., 2015). Also, finding IAA content more concentrated on 25 DAPs in both species indicated an accumulation during zygotic embryogenesis initiation, which is expected to occur on this day. In this respect, Xie et al. (2016) reported that the fertilization process in the ovary was completed after 9 days 
of cross-pollination between $R$. decorum and R. irroratum, in which the cell division was accelerated to cause the fruit to expand rapidly. Previously, it has been reported that IAA regulate zygotic embryo formation in many species. Chen et al. (2010) reported the highest level of IAA in Nicotiana tabacum during proembryo development, which is followed by rapidly forming the young embryos. Moreover, IAA was essential for early Arabidopsis embryo formation as described by Möller and Weijers (2009). Similarly, IAA was an endogenous key factor to asymmetric division of the zygote and root fates at the poles of globular embryos in many plant species (Robert et al., 2013). To confirm this idea, distribution of IAA was studied during microspore embryogenesis in oak trees, barley and rapeseed plants, in which localization of that hormone was clearly found at globular embryo stage (Rodríguez-Sanz et al., 2015).

On the other hand, the growth of fruit inhibited ABA content after cross-pollination in both two fruits in the present paper. It has been suggested that $\mathrm{ABA}$ is a promoter of fruit set and ripening (Leng et al., 2014). Furthermore, Zou et al. (2015) found high ABA level in ovary after selfpollination compared with cross-pollination in Castanea sp., leading to a possible relationship between this hormone and self-incompatibility as also described by other investigators (Zhang et al., 2014). It is worthy to note that ABA can cause flower abscission (Zhang et al., 2014) and it also plays a key role in programmed cell death (Li et al., 2010). Thereupon, our result may confirm the inhibition effect of ABA on biological process in woody plants. ZA level was very low on 0 DAPs, then peaked on 17 DAPs followed by a highly significant decline which peaked again during ovary development, indicating that ZA is needed for both initiation rhododendron fruit. On the contrary, ZA level was low during early stage in date palm fruit (Rastegar et al., 2011). In most cases, it has been reported that ZA content can evidently accelerate the cell division in angiosperms (Xu et al., 2019).

Regarding the investigation on gibberellins, $\mathrm{GA}_{9}, \mathrm{GA}_{19}$ and $\mathrm{GA}_{4}$ may have a key role during ovary growth in $M S$ and $M C$, the highest level of these hormones was found on 17 DAPs. Meanwhile, we found that $\mathrm{GA}_{4}, \mathrm{GA}_{44}$ and $\mathrm{GA}_{20}$ contents peaked on 47 DAPs, indicating their role in fruit formation in Rhododendron. Gibberellins have a capability in the promotion of cell enlargement of fruit (Rastegar et al., 2011). Also, gibberellins have the same pattern of IAA during fruit ripening in which the auxin signaling is involved in GA biosynthesis in the ovule (Gomez et al., 2016). In addition, it has been indicated that Auxin Response Factor (SIARF7) gene has a role in mediating the crosstalk between auxin and GA, as that gene can modify the GA response during early stages of fruit and ovary development (De Jong et al., 2010). Generally, no reports have been studied in EPHs during ovary development in Rhododendron species. A few reports indicated the changes of EPHs during plant development in Rhododendron species, in which most of these investigations studied the importance of EPHs during root development (Elmongy et al., 2018) and floral growth (Meijón et al., 2010). Li et al. (2016) measured GA3, ABA, IAA, and ZA contents during flower development in $R$. simsii, whereas $\mathrm{Wu}$ et al. (2011) studied the levels of the same endogenous hormones in $R$. hybridum. In addition to that, it has been reported that there are clear differences between floral and vegetative buds in azalea plants, with respect to the levels of gibberellins and cytokinins (Meijón et al., 2010).

In China, flower and fruit development in Rhododendron species are always forced by climatic conditions, especially temperature control and light intensity as well as photoperiod (Bhattarai and Upadhyay, 2015). Moreover, it has been shown that $\mathrm{GA}_{3}$, IAA and ZA were enhanced by high temperature and light intensities in Rhododendron hybrids flower bud (Chen and Xian, 2016). In angiosperms, growth of ovary followed by initiation and ripening of fruit is a very critical process due to its tremendous diversity among the different plant species (Skinner et al., 2004). Thus, we cannot determine which one of the studied species has more concentration levels of endogenous hormones. Furthermore, the mechanisms of endogenous hormone regulation are still poorly elucidated. Our results show significant variations between $M S$ and $M C$ although no significant differences were found during ovary growth, pointing to the genotype of the male parents used to pollinate the female $R$. delavayi.

In conclusion, we indicated that there are significant differences in EPHs contents during ovary growth between $M S$ and $M C$ but the pattern during days after cross-pollination is relatively similar. Jasmonate has a major role in fruit formation during days after pollination in our studied species. We suggest that the high concentrations of jasmonic acid are due to low temperature stress facing by rhododendron plants in the forest. The lower endogenous hormones also have a decisive role during ovary development after crosspollination in which high IAA and ABA were probably related to fruit initiation as well as $\mathrm{GA}_{9}, \mathrm{GA}_{19}$, and $\mathrm{GA}_{4}$.

\section{Further work}

To confirm the theory of the effectiveness of the biotic and abiotic stress on the changes in endogenous hormones during fruit development in rhododendron, cold and heat stress gene expression analyses are needed. Also, localization of the enzymes responsible for endogenous hormone syntheses is required to understand their distribution during fruit and ovule development.

\section{Acknowledgments}

This study was supported by the National Natural Science Foundation of China (Grant Nos. 31560225, 31760231, 31760210 and 31760229), Applied Basic Research Project of Yunnan Province (Grant No. 2018BB010), Innovative Talent Development Project of Yunnan Province (Grant No. 2018HB082), and The Talented Young Scientist Program of China (Egypt-17-034).

\section{References}

Baldwin, I.T., Halitschke, R., Paschold, A., Von Dahl, C.C., and Preston, C.A. (2006). Volatile signaling in plant-plant interactions: "Talking trees" in the genomics era. Science 311, 812-815. http://doi. org/10.1126/science.1118446.

Ballaré, C.L. (2011). Jasmonate-induced defenses: A tale of intelligence, collaborators and rascals. Trends in Plant Sci. 16(5), 249-257. http://doi.org/10.1016/j.tplants.2010.12.001.

Barendse, G.W.M., Karssen, C.M., and Koornneef, M. (1991). Role of endogenous gibberellins during fruit and seed development. In Gibberellins, N. Takahashi, B.O. Phinney, and J. MacMillan, eds. (New York, NY: Springer), ISBN 978-1-4612-7754-5. https://doi. org/10.1007/978-1-4612-3002-1_17.

Bharali, S., and Latif Khan, M. (2012). Rhododendrons in Arunachal Pradesh. N. Z. Rhododendron Assoc. Bull., No. 100.

Bhattarai, K.R., and Upadhyay, T. (2015). Rhododendron species richness patterns and impacts of global warming on its distribution in Central Himalayas, Nepal. Botanica Orientalis - J. Plant Sci. 9, 1219. https://doi.org/10.3126/botor.v9i0.21009. 
Chamberlain, D., Hyam, R., Argent, G., Fairweather, G., and Walter, K.S. (1996). The genus Rhododendron: Its classification and synonymy. Royal Botanic Garden Edinburgh. ISBN 187229166X.

Chen, D., Ren, Y., Deng, Y., and Zhao, J. (2010). Auxin polar transport is essential for the development of zygote and embryo in Nicotiana tabacum L. and correlated with ABP1 and PM H+-ATPase activities. J. Exp. Bot. 61(6), 1853-1867. http://doi.org/10.1093/jxb/erq056.

Chen, R., and Xian, X.L. (2016). Effects of different temperatures and light intensities on endogenous hormone in Rhododendron hybrids forcing. Southwest China J. Agric. Sci. 29(10), 2341-2345.

De Jong, K., Ashman, T.L., Collin, C.L., and Laurent, P. (2006). Evolutionary ecology of plant reproductive strategies. Ecology: Ecolog. Soc. of Am. 87(9), 2404. http://doi.org/10.5860/ choice.43-6525.

De Jong, M., Wolters-Arts, M., García-Martínez, J.L., Mariani, C., and Vriezen, W.H. (2010). The Solanum lycopersicum AUXIN RESPONSE FACTOR 7 (SlARF7) mediates cross-talk between auxin and gibberellin signalling during tomato fruit set and development. J. Exp. Bot. 62(2), 617-626. http://doi.org/10.1093/jxb/erq293.

Demsar, J., Curk, T., and Erjavec, A. (2013). Orange: data mining toolbox in Python. J. Machine Learning Res. 14, 5.

De Milleville, R. (2002). The Rhododendrons of Nepal. (Himal Books), 138 pp.

Ding, E.L., Song, Y., Malik, V.S., and Liu, S. (2006). Sex differences of endogenous sex hormones and risk of type 2 diabetes: A systematic review and meta-analysis. JAMA 295(11), 1288-1299. http://doi. org/10.1001/jama.295.11.1288.

Elmongy, M.S., Yan Cao, Zhou, H., and Xia, Y. (2018). Root development enhanced by using indole-3-butyric acid and naphthalene acetic acid and associated biochemical changes of in vitro Azalea microshoots. J. Plant Growth Regul. 37, 813-825. https://doi.org/10.1007/ s00344-017-9776-5.

Ergun, N.S., and Topcuoglu, F. (2002). Auxin (indole-3-acetic acid), gibberellic acid (GA3), abscisic acid (ABA) and cytokinin (zeatin) production by some species of mosses and lichens. Turk. J. Bot. 26, 13-18.

Gomez, M.D., Ventimilla, D., Sacristan, R., and Perez-Amador, M.A. (2016). Gibberellins regulate ovule integument development by interfering with the transcription factor ATS. Plant Physiol. 172(4), 2403-2415. http://doi.org/10.1104/pp.16.01231.

Gram, W.K., and Sork, V.L. (1999). Population density as a predictor of genetic variation for woody plant species. Conserv. Biol. 13(5), 1079-1087. https://doi.org/10.1046/j.1523-1739.1999.98348.x.

Kita, K., Kurashige, Y., Yukawa, T., Nishimuram S., et al. (2005). Intergeneric hybridization between Menziesia and Rhododendron based on molecular phylogenetic data. J. Jap. Soc. Hortic. Sci. 74(1), 51-56. https://doi.org/10.2503/jjshs.74.51.

Kondo, S., and Tomiyama, A. (2000). Changes of endogenous jasmonic acid and methyl jasmonate in apples and sweet cherries during fruit development. J. Am. Soc. Hortic. Sci. 125(3), 282-287. https://doi.org/10.21273/JASHS.125.3.282.

Koornneef, M., Reuling, G., and Karssen, C.M. (1984). The isolation and characterization of abscisic acid-insensitive mutants of Arabidopsis thaliana. Physiol. Plant. 61(3), 377-383. https://doi. org/10.1111/j.1399-3054.1984.tb06343.x.

Kumar, R., Khurana, A., and Sharma, A.K. (2014). Role of plant hormones and their interplay in development and ripening of fleshy fruits. J. Experim. Bot. 65(16), 4561-4575. https://doi.org/10.1093/ jxb/eru277.

Leng, P., Yuan, B., and Guo, Y. (2014). The role of abscisic acid in fruit ripening and responses to abiotic stress. J. Experim. Bot. 65(16), 4577-4588. http://doi.org/10.1093/jxb/eru204.
Li, Z., Jo, J., Jia, J.M., et al. (2010). Caspase-3 activation via mitochondria is required for long-term depression and AMPA receptor internalization. Cell 141(5), 859-871. http://doi.org/10.1016/j.cell.2010.03.053.

Li, Z., Jiang, R., Jin, L., Xu, L., Zhang, M., and Wang, S. (2016). Dynamic changes in endogenous hormones in Rhododendron simsii floral organs. Agric. Biotechnol. 5(5), 19-21.

Liu, L., Wang, Z., Liu, J., et al. (2018). Histological, hormonal and transcriptomic reveal the changes upon gibberellin-induced parthenocarpy in pear fruit. Hortic. Res. 5(1). http://doi. org/10.1038/s41438-017-0012-z.

Liu, Xu, Liao, M.-a., Deng, G.-t., Chen, S.-b., Ren, Y.-j., and Liu, W.-g. (2008). Changes in endogenous hormones and polyamines of fruit during growth and development of pear fruits. World J. Agric. Sci. $4(1), 40-47$.

Luypaert, G., Witters, J., Van Huylenbroeck, J., et al. (2017). Induced expression of selected plant defence related genes in pot azalea, Rhododendron simsii hybrid. Euphytica 213(227). https://doi. org/10.1007/s10681-017-2010-5

Ly, T.T., Nicholas, J.A., Retterath, A., Lim, E.M., Davis, E.A., and Jones, T.W. (2013). Effect of sensor-augmented insulin pump therapy and automated insulin suspension vs standard insulin pump therapy on hypoglycemia in patients with type 1 diabetes: a randomized clinical trial. JAMA 310(12), 1240-1247. http://doi.org/10.1001/ jama.2013.277818.

Ma, Y., Milne, R.I., Zhang, C., and Yang, J. (2010). Unusual patterns of hybridization involving a narrow endemic Rhododendron species (Ericaceae) in Yunnan, China. Am. J. Bot. 97(10), 1749-1757. https:// doi.org/10.3732/ajb.1000018.

Meijón, M., Feito, I., Valledor, L., Rodríguez, R., and Cañal, M.J. (2010). Dynamics of DNA methylation and Histone $\mathrm{H} 4$ acetylation during floral bud differentiation in azalea. BMC Plant Biol. 10(10). http:// doi.org/10.1186/1471-2229-10-10.

Meijón, M., Feito, I., Valledor, L., Santamaría, E., Testillano, P.S., Risuño, M.C., Rodríguez, R., Feito, I., and Cañal, M.J. (2009). Epigenetic characterization of the vegetative and floral stages of azalea buds: dynamics of DNA methylation and histone $\mathrm{H} 4$ acetylation. J. Plant Physiol. 166(15), 1624-1636. https://doi.org/10.1016/j. jplph.2009.04.014.

Möller, B., and Weijers, D. (2009). Auxin control of embryo patterning. Cold Spring Harbor Perspect. Biol. 1(5). http://doi.org/10.1101/ cshperspect.a001545.

Ng, S.-C., and Corlett, R.T. (2000). Genetic variation and structure in six Rhododendron species (Ericaceae) with contrasting local distribution patterns in Hong Kong, China. Molec. Biol. 9(7), 959969. https://doi.org/10.1046/j.1365-294x.2000.00958.x.

Oh, Y., Baldwin, I.T., and Galis, I. (2013). A jasmonate ZIM-domain protein $\mathrm{NaJAZd} \mathrm{regulates} \mathrm{floral} \mathrm{jasmonic} \mathrm{acid} \mathrm{levels} \mathrm{and} \mathrm{counteracts}$ flower abscission in Nicotiana attenuata plants. PLoS One $8(2)$. http://doi.org/10.1371/journal.pone.0057868.

Pattison, R.J., Csukasi, F., Zheng, Y., Fei, Z., Van der Knaap, E., and Catalá, C. (2015). Comprehensive tissue-specific transcriptome analysis reveals distinct regulatory programs during early tomato fruit development. Plant Physiol. 168(4), 1684-1701. http://doi. org/10.1104/pp.15.00287

Perrot-Rechenmann, C. (2010). Cellular responses to auxin: division versus expansion. Cold Spring Harbor Perspect. Biol. 2(5). http:// doi.org/10.1101/cshperspect.a001446.

Rastegar, S., Rahemi, M., and Zargari, H. (2011). Changes in endogenous hormones in fruit during growth and development of date palm fruits. Am.-Euras. J. Agric. \& Environ. Sci. 11(2), 140-148. 
Reinhardt, D., Mandel, T., and Kuhlemeier, C. (2000). Auxin regulates the initiation and radial position of plant lateral organs. Plant Cell 12(4), 507-518. http://doi.org/10.2307/3871065.

Robert, H.S., Grones, P., Stepanova, A.N., Robles, L.M., Lokerse, A.S., Alonso, J.M., Weijers, D., and Friml, J. (2013). Local auxin sources orient the apical-basal axis in Arabidopsis embryos. Current Biol. 23(24), 2506-2512. https://doi.org/10.1016/j.cub.2013.09.039.

Robert, H.S., Khaitova, L.C., Mroue, S., and Benková, E. (2015). The importance of localized auxin production for morphogenesis of reproductive organs and embryos in Arabidopsis. J. Experim. Bot. 66(16), 5029-5042. https://doi.org/10.1093/jxb/erv256.

Rodriguez-Sanz, H., Moreno-Romero, J., Solis, M.T., Kohler, C., Risueno, M.C., and Testillano, P.S. (2014). Changes in histone methylation and acetylation during microspore reprogramming to embryogenesis occur concomitantly with BnHKMT and BnHAT expression and are associated with cell totipotency, proliferation, and differentiation in Brassica napus. Cytogenet. Genome Res. 143(1-3), 209-218. https:// doi.org/10.1159/000365261.

Rodríguez-Sanz, H., Solís, M.T., López, M.F., Gómez-Gadenas, A. Risueño, M.C., and Testillano, P.S. (2015). Auxin biosynthesis, action and transport are involved in stress-induced microspore embryogenesis initiation and progression in Brassica napus L. Plant Cell Physiol. 56, 1401-1417. https://doi.org/10.1093/pcp/pcv058.

Sang, Y.L., Cheng, Z.J., and Zhang, X.S. (2016). Endogenous auxin biosynthesis and de novo root organogenesis. J. Experim. Bot. 67, 4011-4013. http://doi.org/10.1093/jxb/erw250.

Santner, A., and Estelle, M. (2009). Recent advances and emerging trends in plant hormone signalling. Nature 459, 1071-1078. https:// doi.org/10.1038/nature08122.

Sauer, M., Robert, S., and Kleine-Vehn, J. (2013). Auxin: simply complicated. J. Experim. Bot. 64, 2565-2577. http://doi. org/10.1093/jxb/ert139.

Skinner, D.J., Hill, T.A., and Gasser, C.S. (2004). Regulation of ovule development. Plant Cell 16 (suppl. 1), S32-S45. http://doi. org/10.1105/tpc.015933.

Su, Y.H., Liu, Y.B., Bai, B., and Shen, X. (2015). Establishment of embryonic shoot-root axis is involved in auxin and cytokinin response during Arabidopsis somatic embryogenesis. Front. Plant Sci. 5, 792. https://doi.org/10.3389/fpls.2014.00792.

Tamaoki, D., Seo, S., Yamada, S., et al. (2013). Jasmonic acid and salicylic acid activate a common defense system in rice. Plant Signal Behav. 8(6), e24260. http://doi.org/10.4161/psb.24260.

Tank, J.G., Pandya, R.V., and Thaker, V.S. (2015). IAA and zeatin controls cell division and endoreduplication process in quiescent center cells of Allium cepa root. Ind. J. Plant. Physiol. 20(2), 124-129. https://doi.org/10.1007/s40502-015-0148-5.

Tian, H., and Russell, S. (1997). Micromanipulation of male and female gametes of Nicotiana tabacum: I. Isolation of gametes. Plant Cell Rep. 16(8), 555-560. http://doi.org/10.1007/BF01142323.

Wu, Y.Y., Li Bo, Zhu, P., and Hu, H.Y. (2011). Effects of plant growth regulator on flowering and endogenous hormones of Rhododendron hybridum. Acta Hortic. Sinica 38(8), 1565-1571.

Xie, W.J., Wang, J.H., Peng, L.C., Song, J., Lu, L., Xu, H.L., and Li, S.F. (2016). The compatibility and fruit growth dynamics of the cross between Rhododendron decorum and R. irroratum. Acta Agric. Univ. Jiangxiensis 38(1), 90-96.

Xu, Z.L., Chen, Y.C., Gao, M., Wu, L.W., Zhao, G.X., and Wang, Y.D. (2019). Research progress in sex differentiation in angiosperms. Sci. Silvae Sinicae 55(8), 157-169. http://doi:10.11707 /j.1001-7488.20190817.
Yildiz, E., and Kaplankiran, M. (2017). The effect of cross-pollination on fruit set and quality in 'Robinson' and 'Fremont' mandarins. Ege Üniv. Ziraat Fak. Derg. 54(1), 107-112. https://doi.org/10.20289/ zfdergi.388390.

Zha, H.G., Milne, R.I., and Sun, H. (2008). Morphological and molecular evidence of natural hybridization between two distantly related Rhododendron species from the Sino-Himalaya. Bot. J. Linnean Soc. 156(1), 119-129. http://doi.org/10.1111/j.10958339.2007.00752.x.

Zha, H.-G., Milne, R.I., and Sun, H. (2010). Asymmetric hybridization in Rhododendron agastum: A hybrid taxon comprising mainly $\mathrm{F}_{1} \mathrm{~s}$ in Yunnan, China. Ann. Bot. 105(1), 89-100. http://doi.org/10.1093/ aob/mcp267.

Zhang, C.C., Wang, L.Y., Wie, K., et al. (2014). Transcriptome analysis reveals self-incompatibility in the tea plant (Camellia sinensis) might be under gametophytic control. BMC Genomics 17, 359. http://doi. org/10.1186/s12864-016-2703-5.

Zhang, J.L., Zhang, C.Q., Gao, L.M., Yang, J.B., and Li, H.T. (2007). Natural hybridization origin of Rhododendron agastum (Ericaceae) in Yunnan, China: Inferred from morphological and molecular evidence. J. Plant Res. 120, 457-463. http://doi.org/10.1007/ s10265-007-0076-1.

Zhang, S., Huang, L., Yan, A., Liu, Y., Liu, B., Yu, C., Zhang, A., Schiefelbein, J., and Gan, Y. (2016). Multiple phytohormones promote root hair elongation by regulating a similar set of genes in the root epidermis in Arabidopsis. J. Experim. Bot. 67(22), 6363-6372. https://doi. org/10.1093/jxb/erw400.

Zou, F., Guo, S.J., Xiong, H., Peng, J.J., Wang, L.Y., Wang, J., and Xu, C. (2015). Changes of endogenous hormone levels during ovary growth and development after self- and cross-pollination of chestnut (Castanea) cultivar 'Yanshanzaofeng'. Adv. J. Food Sci. Technol. 8(11), 796-801. http://doi.org/10.19026/ajfst.8.1610.

Received: Jan. 10, 2019

Accepted: Oct. 18, 2019

Addresses of authors:

Ahmed Abdalla El-Tantawy ${ }^{1,2}$, Weijia Xie ${ }^{1,3, *}$, Shifeng $\mathrm{Li}^{1}$, Jihua Wang ${ }^{1}$, Lvchun Peng ${ }^{1}$, Jie Song ${ }^{1}$, Lu Zhang ${ }^{1}$, Yanfei Cai ${ }^{1}$ and Xiumei Yang ${ }^{1}$

${ }^{1}$ Flower Research Institute, Yunnan Academy of Agricultural Sciences, Kunming, China

2 Ornamental Horticulture Department, Faculty of Agriculture, Cairo University, Egypt

${ }^{3}$ School of Life Sciences, Center for Plant Disease \& Pest

Biocontrol, Yunnan University, Kunming, China

* Corresponding author; E-mail: weijiax@163.com 